\title{
Design of Fuzzy Tuned PID Controller for Anti Rolling Gyro (ARG) Stabilizer in Ships
}

Muhammad Talha ${ }^{1}$, Furqan Asghar ${ }^{1}$, and Sung Ho Kim ${ }^{2}$

${ }^{1}$ School of Electronics and Information Engineering, Kunsan National University, Gunsan, Korea

${ }^{2}$ Department of Control and Robotics Engineering, Kunsan National University, Gunsan, Korea

\section{ljfis}

Received: Aug. 17, 2017

Revised : Aug. 31, 2017

Accepted: Sep. 13, 2017

Correspondence to: Sung Ho Kim

(shkim@kunsan.ac.kr)

(CThe Korean Institute of Intelligent Systems

CCThis is an Open Access article distributed under the terms of the Creative Commons Attribution Non-Commercial License (http://creativecommons.org/licenses/ by-nc/3.0// which permits unrestricted noncommercial use, distribution, and reproduction in any medium, provided the original work is properly cited.

\begin{abstract}
The Anti Rolling Gyro (ARG) is a roll reduction device for vessels that utilizes gyro torque stabilization. One of the ARG's main features is to reduce ship rolling motion even at zero speed which is impossible by using fin stabilizer. ARG consists of a dedicated spinning wheel that generates gyroscopic forces to reduce the roll motion of ships. The aim of ARG stabilizer is to minimize the effect of external wave disturbances acting on the ship. Two of the most commonly used ARG types in ships are horizontal spin axis type and vertical spin axis type with distinct mathematical modeling and control. Control system design is a key factor to improve stabilizing system performance. However, performance improvement falls short of expectations due to fundamental performance limitations, large variations caused by wave induced roll motion and some practical design complications. This paper proposed fuzzy tuned PID control strategy for ARG stabilizers and present comparison between commonly used PID control technique, fuzzy control, and proposed fuzzy tuned PID control technique. These methods have been implemented and validated via simulation approach for horizontal spinning axis ARG. Simulation results prove the effectiveness and supremacy of proposed technique over previously used methods.
\end{abstract}

Keywords: Anti Rolling Gyro (ARG), Ship motion control, Roll damping in ships, Gyro stabilizer

\section{Introduction}

Ship rolling motion reduction induced by waves is a matter of paramount importance in marine vessels since it affects crew, passengers and onboard equipment operations. Gyroscopic stabilizers are being used since decades to minimize wave induced disturbances, either it is related to small size vessels or huge cargo ships. Since the gyro stabilizers have a broad implementation in various vehicles and robots, its basic construction in all cases is almost same. Generally, a gyrostabilizer consists of one or more dedicated spinning wheels used to counteract roll excitation forces by their gyroscopic effect. Figure 1 shows horizontal and vertical spin axis Anti Rolling Gyro (ARG) stabilizer mounted on a vessel. The gyroscope has a long history of application in engineering design due to two properties: rigidity and precession. Rigidity is an important characteristic of gyroscopes where a spinning gyroscope will maintain its orientation in space. These gyroscopic properties are utilized in various applications such as navigation systems, actuator and passive stabilization systems used in torpedoes and ships. ARG systems use conservation of angular momentum to stabilize unstable bodies by functioning as an actuator using the phenomenon of gyroscopic precession. 


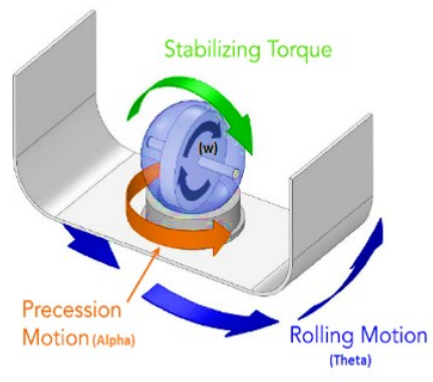

Horizontal Spin Axis

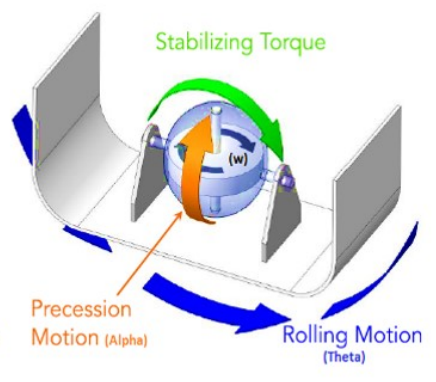

Vertical Spin Axis
Figure 1. ARG types.

If a flywheel is spinning about the $\omega$ axis and an external disturbance is applied about the $\theta$ axis, i.e., wave roll disturbance. In this case, if it has sufficient angular momentum, it will stay horizontal and begin to spin around $\alpha$ axis. This spin about $\alpha$ axis is called precession and this type of ARG is called passive control ARG [1]. The natural precession phenomenon is enhanced by using some control techniques (PID, fuzzy logic) to provide faster stabilizing response known as active stabilization system. Precession motion of gyrostabilizer provides stabilization torque which is transferred to the ship via the frame. In this paper, fuzzy tuned PID controller based active precession control system is proposed and comparison study of proposed technique with previously used techniques (PID, fuzzy logic) is discussed and tested by implementation in Matlab/Simulink. Furthermore, their benefits and drawbacks are discussed in detail.

\section{Dynamics of Ship}

Ship dynamics can be described using following general model structure [2].

$$
\begin{aligned}
& \dot{\boldsymbol{\eta}}=\boldsymbol{J}(\boldsymbol{\eta}) \boldsymbol{v} \\
& \boldsymbol{M}_{R B} \dot{\boldsymbol{v}}+\boldsymbol{C}_{R B}(\boldsymbol{v}) \boldsymbol{v} \doteq \boldsymbol{\tau}_{h}+\boldsymbol{\tau}_{c}+\boldsymbol{\tau}_{d} .
\end{aligned}
$$

Here, the vector variables $\boldsymbol{\eta}$ and $\boldsymbol{v}$ represent the generalized displacement and body-fixed velocities, and $\tau_{h}, \tau_{c}$, and $\tau_{d}$ represent the hydrodynamics, control and disturbance forces, respectively.

To describe the vessel motion, a reference frame $\{b\}$ is considered fixed to the vessel at the point $o_{b}$ and a local geographical frame $\{n\}$ is considered fixed to the mean water level at the location $o_{n}$. These reference frames are illustrated in Figure 2.

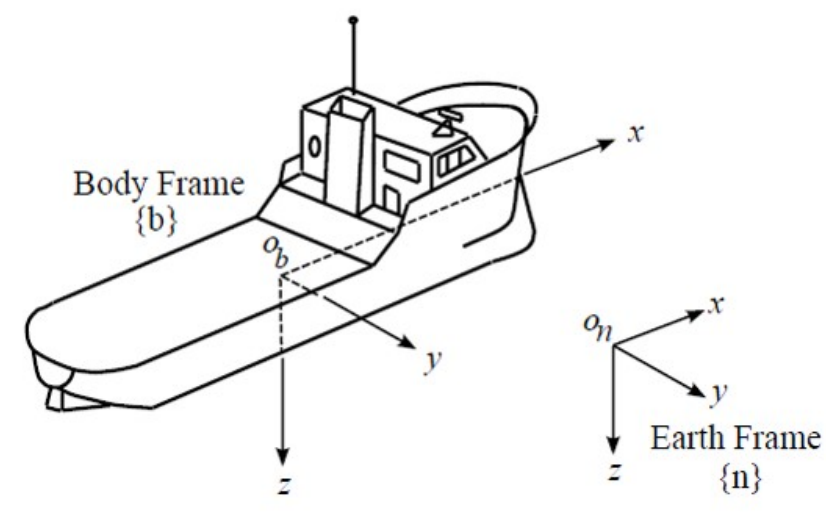

Figure 2. Ship frame and motion description.

The generalized position and velocity vectors are given by

$$
\begin{aligned}
& \boldsymbol{\eta}=[n, e, d, \phi, \theta, \psi], \\
& \boldsymbol{v}=[u, v, w, p, q, r]^{T} .
\end{aligned}
$$

The components of $\boldsymbol{\eta}$ are the north, east and down positions of $\mathrm{o}_{b}$ with respect to $\{\mathrm{n}\}$ and the Euler angles $\phi$ (roll), $\theta$ (pitch), and $\psi$ (yaw), which takes $\{\mathrm{n}\}$ into the orientation of $\{\mathrm{b}\}$. The components of $v$ are the linear velocities expressed in the body-fixed frame, namely $u$-surge, $v$-sway, and $w$-heave. The components of angular velocity vector expressed in the body-fixed frame are the $p$ (roll rate), $q$ (pitch rate) and $r$ (yaw rate). The generalized force vectors in the body-fixed frame are given by

$$
\boldsymbol{\tau}_{i}=\left[X_{i}, Y_{i}, Z_{i}, K_{i}, M_{i}, N_{i}\right]^{T},
$$

Here, $X_{i}$ is the surge force, $Y_{i}$ is the sway force, $Z_{i}$ is the heave force, $K_{i}$ is the roll moment, $M_{i}$ is the pitch moment and $N_{i}$ is the yaw moment. All the moments are taken about $o_{b}$.

The kinematic transformation $\mathbf{J}(\boldsymbol{\eta})$ relates the body-fixed velocities to the time derivative in $\{n\}$ of the generalized positions, which gives the vessel trajectory. The matrix $\mathbf{M}_{R B}$ in Eq. (2) is the rigid-body mass matrix and $\mathbf{C}_{R B}(\boldsymbol{v})$ is the Corioliscentripetal matrix. The term $\mathbf{C}_{R B}(\boldsymbol{v}) \boldsymbol{v}$ in Eq. (2) appears as a consequence of expressing the velocity vector in a rotating (non-inertial) frame [3-5].

The study of roll motion dynamics for control system design is normally done in terms of either one or four degrees of freedom (DOF) models. The choice between models of different complexity depends on the type of motion control system considered and the conditions under which the design is to be 
performed. There may be cases where a full 6DOF model is required. In this paper, we just need 1DOF model (ship roll) to implement ARG system.

\subsection{One Degree of Freedom Model}

For a 1DOF model in a roll, the model representation in Eqs. (11) and (2) can be reduced to the following form.

$$
\begin{aligned}
& \dot{\phi}=p, \\
& \boldsymbol{I}_{x x} \dot{p}=\boldsymbol{K}_{h}+\boldsymbol{K}_{c}+\boldsymbol{K}_{d},
\end{aligned}
$$

where $\boldsymbol{I}_{x x}$ is the rigid-body moment of inertia about the $x$-axis of $\{b\}$. This model assumes that the formulation is at the center of the roll. The hydrodynamic forces can be approximated by the following parametric model:

$$
\boldsymbol{K}_{h} \approx \boldsymbol{K}_{\dot{p}} \dot{p}+\boldsymbol{K}_{p} p+\boldsymbol{K}_{p|p|} p|p|+\boldsymbol{K}(\phi) .
$$

The first term on the right-hand side of Eq. (8) represents a hydrodynamic moment in roll due to a pressure change that is proportional to the roll accelerations and the coefficient $\boldsymbol{K}_{\dot{p}}$ is called roll added mass. The second term is a damping term which captures forces due to wave making, linear skin friction and the coefficient $K_{p}$ is called a linear damping coefficient. The third term is a nonlinear damping term which captures forces due to viscous effects like nonlinear skin friction and eddy making due to flow separation. The last term is a restoring term due to gravity and buoyancy, which for some vessels a linear approximation often suffice:

$$
\boldsymbol{K}(\phi) \approx \boldsymbol{K}_{\phi} \phi, \boldsymbol{K}_{\phi}=\rho g \nabla G M t
$$

where $\rho$ is the water density, gis the acceleration of gravity, $\nabla$ is the vessel displaced volume and $G M t$ is the transverse metacentric height [6, 7].

The coefficients in the hydrodynamic model in Eq. 8) change with the forward speed of the vessel $U$. This can be represented in the model by adding terms, for example

$$
\boldsymbol{K}_{h} \approx \boldsymbol{K}_{h 0}+\boldsymbol{K}_{h U}
$$

where,

$$
\begin{aligned}
& \boldsymbol{K}_{h 0}=\boldsymbol{K}_{\dot{p}} \dot{p}+\boldsymbol{K}_{p} p+\boldsymbol{K}_{p|p|} p|p|+\boldsymbol{K}(\phi), \\
& \boldsymbol{K}_{h U}=\boldsymbol{K}_{U p} \boldsymbol{U} p+\boldsymbol{K}_{\phi U^{2}} \phi \boldsymbol{U}^{2} .
\end{aligned}
$$

As the forward speed changes, so do the trim of the vessel, which can be related to the steady state pitch angle. The trim affects not only the damping but also the restoring term as shown in Eq. 9). Some vessels have trim tabs (see Figure 2) which are used to correct the trim at different cruise speeds as well as to reduce the hydrodynamic resistance and likewise fuel consumption. The use of trim tabs modifies the restoring coefficient in Eq. 99.

\section{Ocean Surface Waves}

Ocean surface waves cause periodic loads on all sorts of manmade structures in the sea. It does not matter whether these structures are fixed, floating or sailing and on the surface or deeper in the sea. To understand these loads, a good understanding of the physics of water waves is necessary. Ocean surface waves are generally distinguished in two states: sea or wind waves. When the waves are being worked on by the wind that raised them and swell or they have escaped the influence of the generating wind. Sea is usually of the shorter period (higher frequency) then swell. A period of about 10 seconds may be taken as separating the sea from swell; although one must allow for considerable overlap [9]. Sea wave is shorter in length, steeper, more rugged and more confused than a swell wave. Since wind-generated waves have their origin in the wind (which is proverbially changeable), these waves change too with the wind, varying both seasonally and regionally.

The velocity of idealized traveling waves on the ocean is wavelength dependent and for shallow enough depths, it also depends upon the depth of the water. The simplest propagating wave of unchanging form is a sine wave. A sine wave with water surface elevation $\eta(x, t)$ is given by

$$
\eta(x, t)=\operatorname{asin}(\theta(x, t)) .
$$

Here, $\mathrm{a}$ is the amplitude (in meters) and $\theta=\theta(x, t)$ is the phase function (in radians), depending on the horizontal position ( $x$, in meters) and time ( $t$, in seconds)

$$
\theta=2 \pi\left(\frac{x}{\lambda}-\frac{t}{T}\right)=k x-w t
$$

where $k=\frac{2 \pi}{\lambda}, w=\frac{2 \pi}{T}, \lambda$ is the wavelength (in meters), $T$ is the period (in seconds), $k$ is the wavenumber (in radians per meter) and $\omega$ is the angular frequency (in radians per second). The wave speed relationship is

$$
V=\sqrt{\frac{g \lambda}{2 \pi} \tanh \left(2 \pi \frac{d}{\lambda}\right)}
$$


where $\lambda$ is wavelength, $d$ is depth and $g$ are gravitational acceleration. Wave height directly depends on wind speed and duration. A 60-knot storm lasting for 10 hours makes $15 \mathrm{~m}$ high waves in open water. Ship roll angle relies upon wave height, higher wave amplitude uplifts the ship roll angle. Just in moderate seas, even very large vessels roll to an angle of $10^{\circ}$. On rare occasions, rolling angles may reach $45^{\circ}$ and above. In this paper, $50^{\circ}$ of roll motion is applied for testing the system response under severe roll angle scenario.

\section{Anti Rolling Gyro (ARG)}

The ARG is rolling reduction device, which is used to compensate wave roll motion of all types of vessels from small, medium to fast moving battle ships. The gyro stabilizes the boat through the energy it creates by spinning a flywheel at high revolutions per minute. The subsequent angular momentum or stabilizing power is determined by the weight, diameter, and RPM of the flywheel measured in Newton meter $(\mathrm{Nm})$ torque. The output rating in $\mathrm{Nm}$ is the amount of power this unit is capable of generating to stabilize the boat. The more output, the more anti-rolling torque generated by the gyro to stabilize the boat.

\subsection{Anti Rolling Gyro Model}

First experimental gyros were developed in the late 1860's and into the early 1900's. Several large ships used this technology including USS Henderson, i.e., a military cargo ship in 1917 with two 25-ton units and an Italian cruise liner utilized three large units in 1930. The cost and weight of the system were prohibitive and other forms of stabilization became more readily available. External fin stabilization which used the speed of the vessel to help create anti-roll stabilization became more popular. With the passage of time, new researches were carried out for improvement in ARG system for easy availability and make it more effective. Mitsubishi Heavy Industries (MHI) is considering and developing ARG systems since 1990. In 2004, MHI formed an exclusive agreement for the sale of ARG devices for vessels with an Italian yacht manufacturer and has since produced and sold them in Japan, the United States, and Asian markets in addition to the main European market.

ARG construction is similar to ordinary gyro stabilizers with slight changes. The ARG has a high-speed spinning flywheel that is supported by a gimbal. When the gimbal is rolled, i.e., an angular velocity is applied to the gimbal, flywheel generates a gyro force in the direction perpendicular to the angular

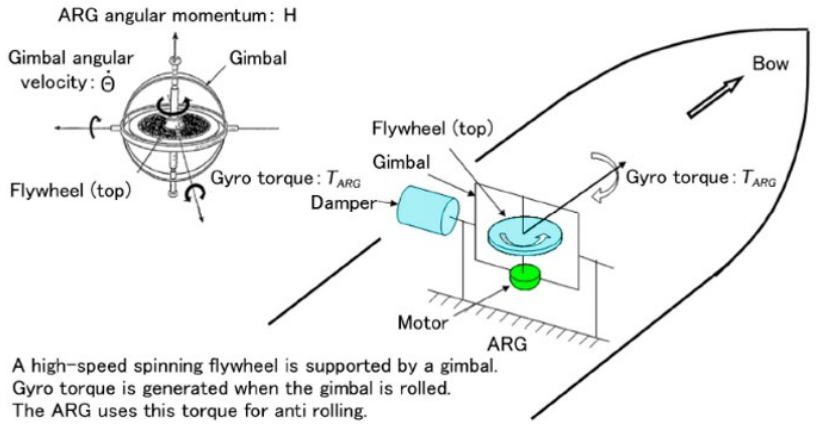

Figure 3. ARG working principle.

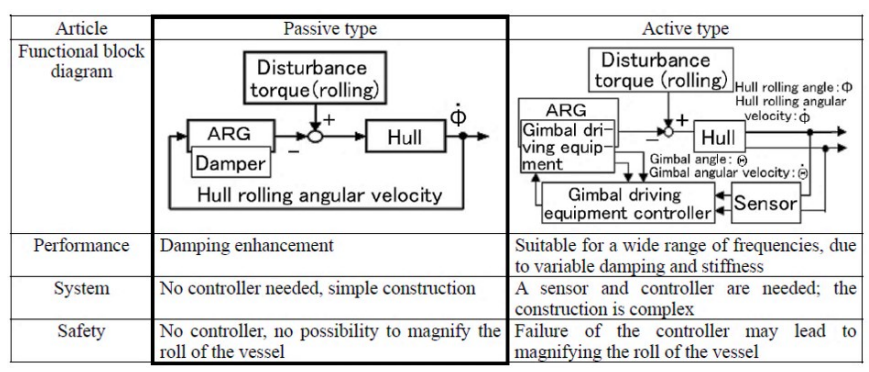

Figure 4. ARG control techniques.

velocity. The damper is used to control the precession angle to its maximum value and apply breaks in case of turning the ship [10]. ARG working principle is shown in Figure 3. This figure shows working principle of two basic types of ARG's depending upon the construction, i.e., horizontal spin axis and vertical spin axis as shown in Figure 1.

In horizontal spin axis, flywheel spinning axis and precession motion are in parallel with vessel frame and vice versa in vertical spin axis ARG. Also, ARG has two types with respect to control, i.e., passive control and active control. Function block diagram for both techniques is shown in Figure 4. As a passive device, it detects the rolling angular velocity of the vessel using the gyro effect and controls the gimbal angular velocity using an energy absorber (damper) [11]. Active control ARG works with a couple of sensors where control system sensors are implemented to measure hull roll angle and hull roll angular velocity. These sensors parameters are fed back to the controller which controls the gimbal angle and gimbal angular velocity to minimize disturbance more effectively.

\subsection{Anti Rolling Gyro Operation}

In ARG, precession motion is the oscillation back and forth through a maximum of $\pm 70^{\circ}$ in the vessel's pitching axis. Precession rate is the rotational speed of this oscillation. Angular 


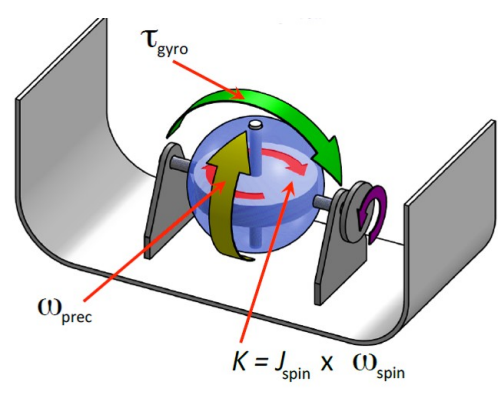

Figure 5. Basic working principle of ARG.

momentum is the rotational inertia of a body multiplied by the speed of rotation. This rotation is analog to linear momentum where the momentum is equal to the mass of the body multiplied by its speed of movement. Any type of induced wave disturbance produces precession motion and this precession motion creates gyro torque. Gyro torque produced can be written in following mathematical form.

$$
\tau_{\text {gyro }}=J_{\text {spin }} \times \omega_{\text {spin }} \times \omega_{\text {prec }},
$$

where $\tau_{\text {gyro }}$ gyro is torque (in Nm), $J_{\text {spin }}$ is flywheel rotational moment of inertia about spin angular velocity $\left(\right.$ in $\left.\mathrm{kg} \cdot \mathrm{m}^{2}\right), \omega_{\text {spin }}$ is spin angular velocity of flywheel (in $\mathrm{rad} / \mathrm{s}$ ) and $\omega_{\text {prec }}$ is precession angular velocity or precession rate (in $\mathrm{rad} / \mathrm{s}$ ).

When the spinning axis is rotated away from vertical in the direction of $\omega_{\text {prec }}$, most of the gyro torque is stabilization torque, but a component of yaw torque is also generated. But its effect on steering of vessel in negligible as damping and inertia of yawing axis is much higher than in the rolling axis. This stabilization of rolling motion will let vessel tracks much better in waves.

$$
\begin{aligned}
& \tau_{\text {stabilization }}=\tau_{\text {gyro }} * \sin \alpha, \\
& \tau_{\text {yaw }}=\tau_{\text {gyro }} * \cos \alpha .
\end{aligned}
$$

As we know, the stabilizing torque for both horizontal and vertical spin axis gyros is equal to the angular momentum multiplied by the precession rate and cosine of the precession angle as shown in Eq. (17). For this, we need to find out input terms of this equation. Angular momentum can be calculated as Eq. 19.

$$
K=J_{S} * \omega_{S}
$$

Here, $k$ represents angular momentum, $J_{S}$ is flywheel rotational inertia in spin axis and $\omega_{s}$ is flywheel spin speed in rad/s.
For precession motion, we can assume it as roughly sinusoidal in nature by considering it proportional to the vessel rolling motion as in Eq. 20].

$$
\omega_{\text {prec }}=\dot{\alpha}_{\max }=\alpha_{\text {range }} * \omega_{\text {roll }}
$$

Here $\omega_{\text {prec }}\left(\alpha_{\max }\right)$ is maximum precession rate whereas $\alpha_{\text {range }}$ and $\omega_{\text {roll }}$ represent precession range and oscillating precession motion frequency, respectively. As it is clear from above equations, gyro torque acts in the plane of both spinning and precession axis. Therefore, when the precession angle is away from vertical, the gyro torque work as stabilizing torque and non-productive torque act on vessel yaw axis but it has no negative effect as inertia and damping of the vessel in yaw axis too high. Furthermore, this yaw component approaches to zero as the precession angle increases towards maximum. This happens because to stop precessing at its maximum range, precession must decelerate and approach zero as it approaches maximum travel. At this point, gyro torque and its raw component are zero. This combination of diminishing amplitude of gyro torque as precession angle reaches to maximum and limiting the range to $\pm 70^{\circ}$ enables the majority of gyro torque to produce stabilizing torque as shown in Eq. (17). Gyro torque can be represented as Eq. 21.

$$
\tau_{\text {gyro }}=K * \omega_{p}
$$

Here $K$ is angular momentum and $\omega_{p}$ is precession rate in $\mathrm{rad} / \mathrm{s}$.

\section{Anti Rolling Gyro Controller}

As mentioned before in Section 4.1, there are two types of controlling techniques for ARG used in ships, i.e., active and passive types as shown in Figure 4. In the passive type of control, there is no control mechanism except the ARG moves due to its natural precession according to rolling angular velocity and angle direction of the ship. Even though this process decreases the rolling effect but gyro stabilizer efficiency can be improved by using some controller to manage the gimbal angular velocity according to precession angle. Therefore, active type controllers use various types of control systems to make ARG response effective in stabilizing the ship. We implement three different types of controls (PID, fuzzy, and fuzzy tuned PID) in active type controller and comparative study is carried out to find out the control system with better and fast response as discussed briefly in below sub-headings. Block diagram of the whole system is shown in Figure 6. 


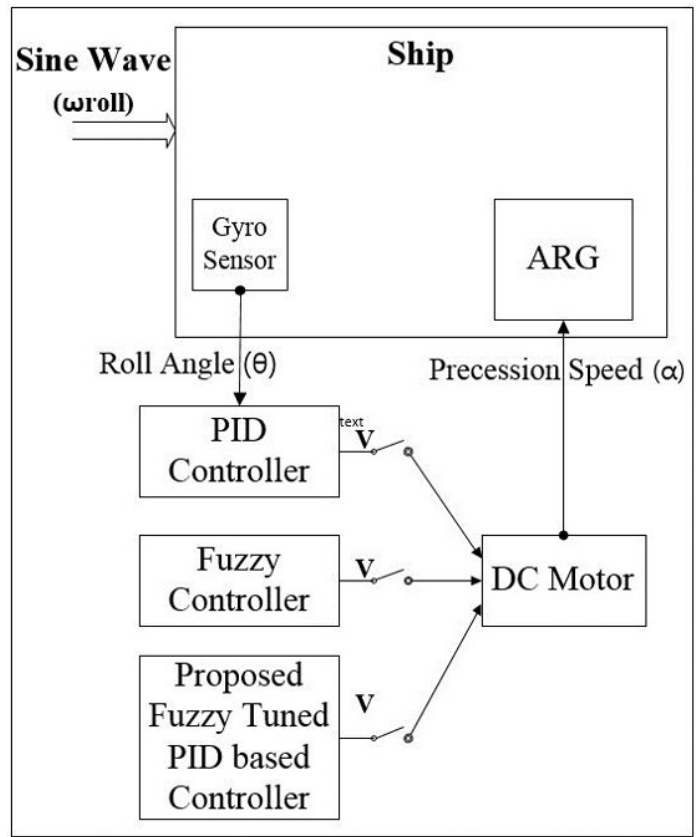

Figure 6. ARG stabilizer control block diagram with various control techniques.

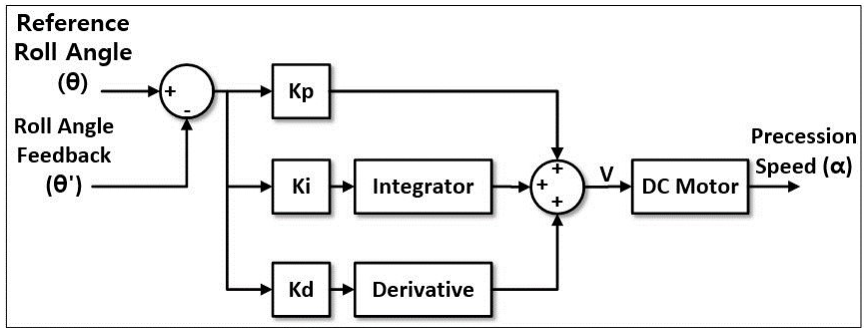

Figure 7. PID controller block diagram.

\subsection{PID Controller for Active Type ARG Stabilizer}

As with any ship roll stabilization system, the primary objective is to reduce roll motion. When water waves hit the vessel bottom, it generates roll motion of the ship. To decrease that roll motion, we used PID controller that can detect roll angle from gyro sensor and control the ARG's precession speed generated by dc motor. This roll angle will be again feedback to PID controller which will keep decreasing the rolling motion of ship by controlling ARG precession speed and try to stabilize ship to best limit possible according to PID gains. Block diagram of PID controller for ARG stabilizer is shown in Figure 7.

But the problem while using PID controller is its fix gains. Due to these fix gains, initial fluctuation for stabilizing rolling motion is high which can have a negative effect on crew or ship's operating equipment.

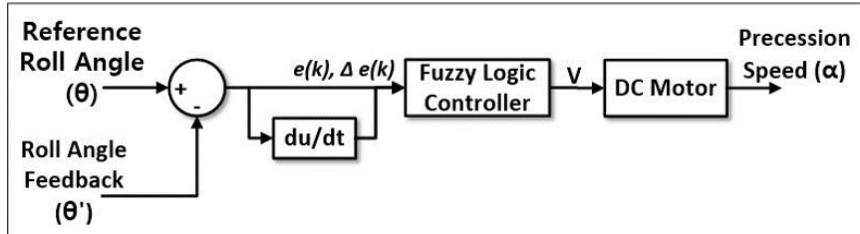

Figure 8. Fuzzy logic based controller block diagram.

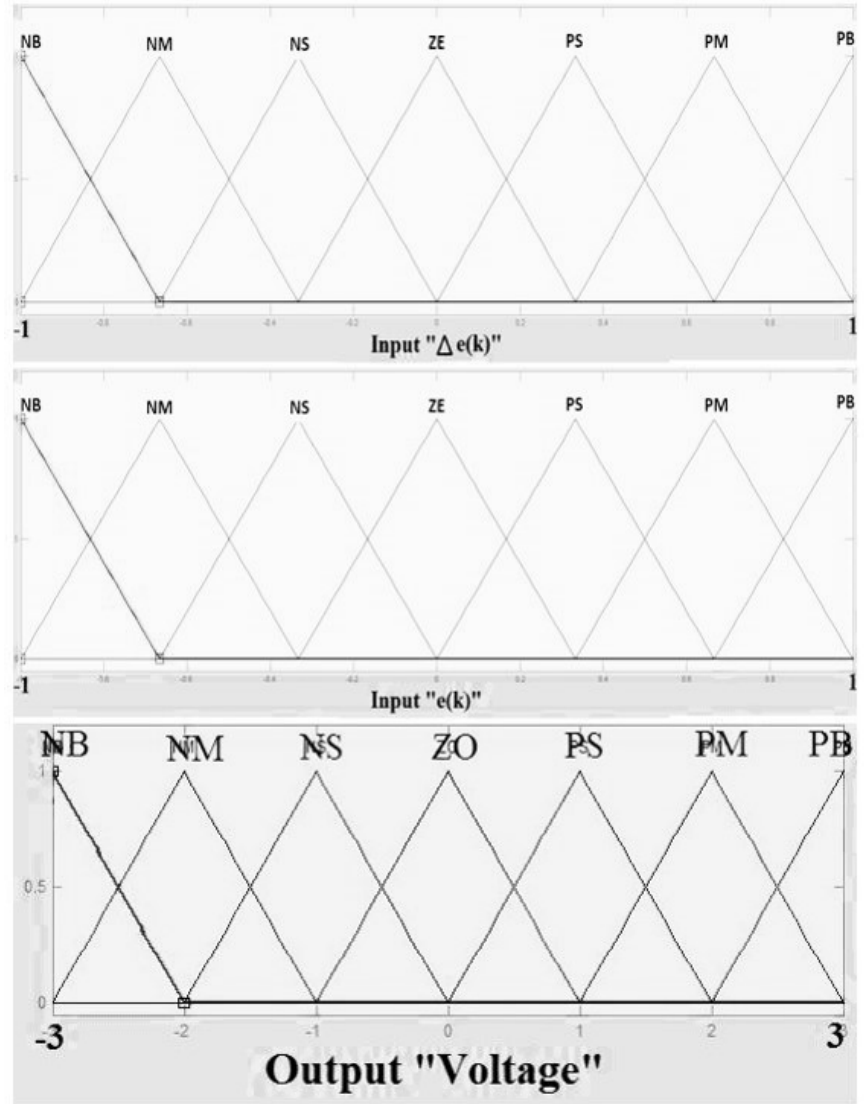

Figure 9. Fuzzy logic input and output membership functions.

\subsection{Fuzzy Logic Controller for Active Type ARG Stabi- lizer}

In this type of controller as shown in Figure 8, the fuzzy logic system is used to generate gains instead of PID to avoid high fluctuations in the start. Here, the fuzzy logic system takes change and rate of change of roll angle as input and control the DC motor voltage which eventually helps to maintain ARG precession speed to stabilize the ship.

Fuzzy logic based controller help to decrease the initial fluctuations but ship's roll angle settling time is quite high which directly affects the system efficiency.

Fuzzy logic consists of two input variables: $e(k)$ and $\Delta e(k)$ and one output variable, i.e., voltage. The membership functions are shown in Figure 9. Furthermore, rule base for voltages 
Table 1. Rule base for voltage

\begin{tabular}{cccccccc}
\hline$\Delta e(k) / e(k)$ & NB & NM & NS & ZE & PS & PM & PB \\
\hline NB & PB & PB & PM & PM & PS & ZE & ZE \\
NM & PB & PB & PM & PS & PS & ZE & NS \\
NS & PM & PM & PM & PS & ZE & NS & NS \\
ZE & PM & PM & PS & ZE & NS & NM & NM \\
PS & PS & PS & ZE & NS & NS & NM & NM \\
PM & PS & ZE & NS & NM & NM & NM & NB \\
PB & ZE & ZE & NM & NM & NM & NB & NB \\
\hline
\end{tabular}

Table 2. Rule base used for $K_{p}$

\begin{tabular}{cccccccc}
\hline$\Delta e(k) / e(k)$ & $\mathrm{NB}$ & $\mathrm{NM}$ & $\mathrm{NS}$ & $\mathrm{ZE}$ & $\mathrm{PS}$ & $\mathrm{PM}$ & $\mathrm{PB}$ \\
\hline $\mathrm{NB}$ & $\mathrm{B}$ & $\mathrm{B}$ & $\mathrm{B}$ & $\mathrm{B}$ & $\mathrm{B}$ & $\mathrm{B}$ & $\mathrm{B}$ \\
$\mathrm{NM}$ & $\mathrm{S}$ & $\mathrm{B}$ & $\mathrm{B}$ & $\mathrm{B}$ & $\mathrm{B}$ & $\mathrm{B}$ & $\mathrm{S}$ \\
$\mathrm{NS}$ & $\mathrm{S}$ & $\mathrm{S}$ & $\mathrm{B}$ & $\mathrm{B}$ & $\mathrm{B}$ & $\mathrm{S}$ & $\mathrm{S}$ \\
$\mathrm{ZE}$ & $\mathrm{S}$ & $\mathrm{S}$ & $\mathrm{S}$ & $\mathrm{B}$ & $\mathrm{S}$ & $\mathrm{S}$ & $\mathrm{S}$ \\
$\mathrm{PS}$ & $\mathrm{S}$ & $\mathrm{S}$ & $\mathrm{B}$ & $\mathrm{B}$ & $\mathrm{B}$ & $\mathrm{S}$ & $\mathrm{S}$ \\
$\mathrm{PM}$ & $\mathrm{S}$ & $\mathrm{B}$ & $\mathrm{B}$ & $\mathrm{B}$ & $\mathrm{B}$ & $\mathrm{B}$ & $\mathrm{S}$ \\
$\mathrm{PB}$ & $\mathrm{B}$ & $\mathrm{B}$ & $\mathrm{B}$ & $\mathrm{B}$ & $\mathrm{B}$ & $\mathrm{B}$ & $\mathrm{B}$ \\
\hline
\end{tabular}

Table 3. Rule base used for $K_{d}$

\begin{tabular}{cccccccc}
\hline$\Delta e(k) / e(k)$ & $\mathrm{NB}$ & $\mathrm{NM}$ & $\mathrm{NS}$ & $\mathrm{ZE}$ & $\mathrm{PS}$ & $\mathrm{PM}$ & $\mathrm{PB}$ \\
\hline $\mathrm{NB}$ & $\mathrm{S}$ & $\mathrm{S}$ & $\mathrm{S}$ & $\mathrm{S}$ & $\mathrm{S}$ & $\mathrm{S}$ & $\mathrm{S}$ \\
$\mathrm{NM}$ & $\mathrm{B}$ & $\mathrm{B}$ & $\mathrm{S}$ & $\mathrm{S}$ & $\mathrm{S}$ & $\mathrm{B}$ & $\mathrm{B}$ \\
$\mathrm{NS}$ & $\mathrm{B}$ & $\mathrm{B}$ & $\mathrm{B}$ & $\mathrm{S}$ & $\mathrm{B}$ & $\mathrm{B}$ & $\mathrm{B}$ \\
$\mathrm{ZE}$ & $\mathrm{B}$ & $\mathrm{B}$ & $\mathrm{B}$ & $\mathrm{B}$ & $\mathrm{B}$ & $\mathrm{B}$ & $\mathrm{B}$ \\
$\mathrm{PS}$ & $\mathrm{B}$ & $\mathrm{B}$ & $\mathrm{B}$ & $\mathrm{S}$ & $\mathrm{B}$ & $\mathrm{B}$ & $\mathrm{B}$ \\
$\mathrm{PM}$ & $\mathrm{B}$ & $\mathrm{B}$ & $\mathrm{S}$ & $\mathrm{S}$ & $\mathrm{S}$ & $\mathrm{B}$ & $\mathrm{B}$ \\
$\mathrm{PB}$ & $\mathrm{S}$ & $\mathrm{S}$ & $\mathrm{S}$ & $\mathrm{S}$ & $\mathrm{S}$ & $\mathrm{S}$ & $\mathrm{S}$ \\
\hline
\end{tabular}

Table 4. Rule base used for $\alpha$

\begin{tabular}{cccccccc}
\hline$\Delta e(k) / e(k)$ & NB & NM & NS & ZE & PS & PM & PB \\
\hline NB & 2 & 2 & 2 & 2 & 2 & 2 & 2 \\
NM & 3 & 3 & 2 & 2 & 2 & 3 & 3 \\
NS & 4 & 3 & 3 & 2 & 3 & 3 & 4 \\
ZE & 5 & 4 & 3 & 3 & 3 & 4 & 5 \\
PS & 4 & 3 & 3 & 2 & 3 & 3 & 4 \\
PM & 3 & 3 & 2 & 2 & 2 & 3 & 3 \\
PB & 2 & 2 & 2 & 2 & 2 & 2 & 2 \\
\hline
\end{tabular}

is shown in Table 1.

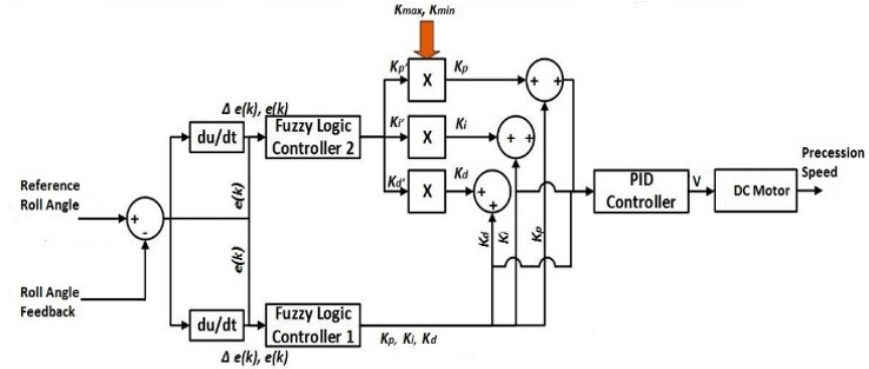

Figure 10. Proposed fuzzy logic tuned PID controller block diagram.

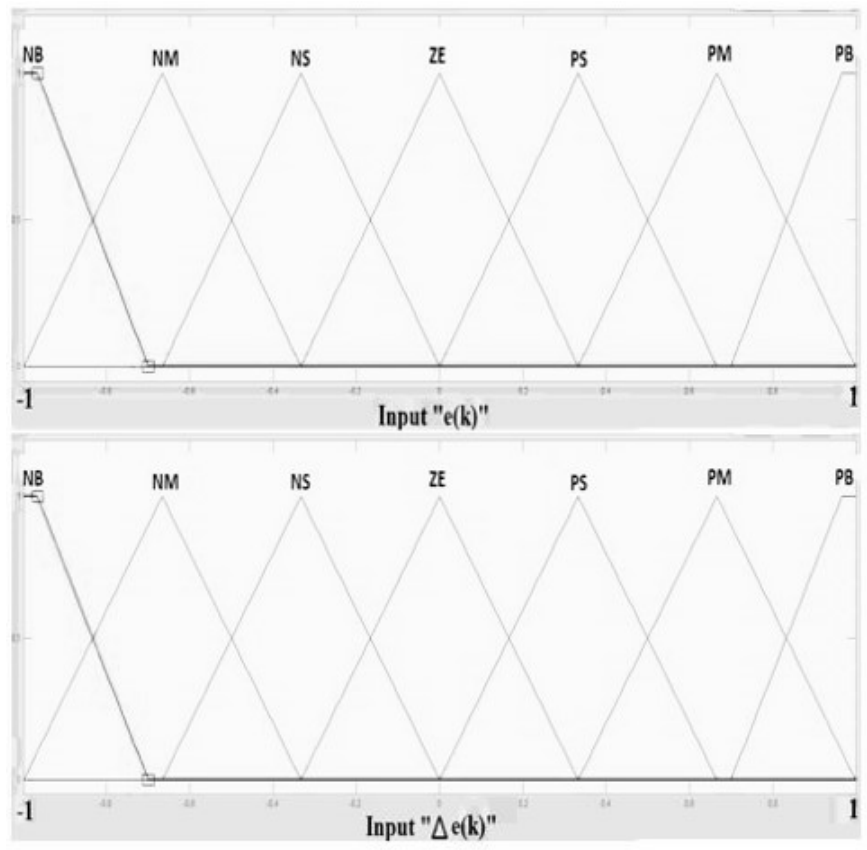

Figure 11. Fuzzy logic tuned PID input membership functions.

\subsection{Proposed Fuzzy Tuned PID Controller for Active Type ARG Stabilizer}

In this proposed type of controller, the fuzzy logic technique is used to control the ARG operation. Fuzzy logic works as gain tuner for PID controller by calculating the $K_{P}, K_{i}, K_{d}$. Comparing with the previous types, the proposed controller works on the base of continuous estimation of rolling angle under changing system conditions and do not require any online tuning methods. Figure 10 shows the proposed control scheme.

The transfer function and discrete time equivalent expression for PID controller are given in Eqs. 22, and 23) as:

$$
G(s)=K_{p}+K_{i} / s+K_{d} s,
$$




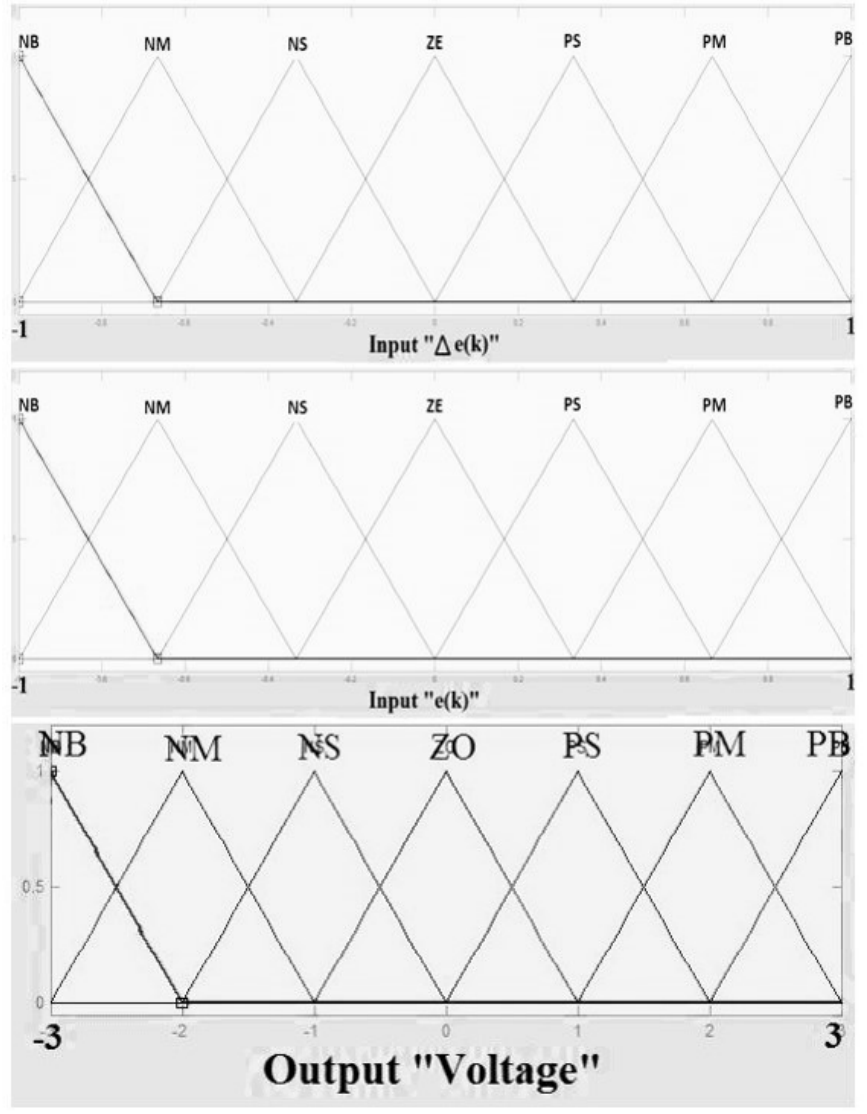

Figure 12. Fuzzy logic tuned PID output membership functions.

$$
u(k)=K_{p} e(k)+K_{i} T_{s} \sum_{i=1}^{n} e(i)+\frac{K_{d}}{T_{s}} \Delta e(k),
$$

where $K_{P}, K_{i}$ and $K_{d}$ are proportional, integral and derivative gains. Also, $T_{i}=K_{p} / K_{i}$ and $T_{d}=K_{d} / K_{p}$.

$T_{i}$ and $T_{d}$ are the integral and derivative time constants, respectively. $T_{s}$ is the sampling time of the controller.

$K_{p}$ and $K_{d}$ are normalized as:

$$
\begin{aligned}
K_{p}^{\prime} & =\frac{K_{p}-K_{p \cdot \min }}{K_{p \cdot \max }-K_{p \cdot \min }}, \\
K_{d}^{\prime} & =\frac{K_{d}-K_{d \cdot \min }}{K_{d \cdot \max }-K_{d \cdot \min }} .
\end{aligned}
$$

Therefore, integral time constant w.r.t derivative time constant and integral time can be calculated as Eq. 26) and Eq. 27), respectively:

$$
\begin{aligned}
& T_{i}=\alpha T_{d}, \\
& K_{i=\frac{K_{p}}{\left(\alpha T_{d}\right)}}=\frac{K_{p}^{2}}{\left(\alpha K_{d}\right)} .
\end{aligned}
$$

From Eq. 24) and Eq. 26);

$$
\begin{aligned}
K_{p} & =\left(K_{p \cdot \max }-K_{p \cdot \min }\right) K_{p}^{\prime}+K_{p \cdot \min } \\
K_{d} & =\left(K_{d . \max }-K_{d . \min }\right) K_{d}^{\prime}+K_{d . \min } .
\end{aligned}
$$

Two input variables $e(k)$ and $\Delta e(k)$, three output variables $K_{P}, K_{d}$ and $\alpha$ are used. Output variable $\alpha$ is used as a fuzzy number which has a singleton membership function. These membership functions are shown in Figures 11 and 12; whereas the rule base for $K_{p}, K_{d}$ and $\alpha$ are shown in Tables 2, 3, and 4, respectively.

\section{Simulation Studies}

Ship dynamics, ocean surface waves effect, ARG design, operation, and control techniques are briefly discussed in Sections 2-5. Now the MATLAB/Simulink study is examined in this section. Simulation diagram of ARG stabilization system with various control techniques is shown in Figure 13.

As we can see, all three controllers explained above are implemented in Simulink to get the system response to verifying proposed control technique supremacy over the previous control systems. System response while stabilizing ship using ARG system is shown in Figure 14.

In PID controller based ARG stabilization, initial gains are fixed due to which big fluctuations can be observed in the start. In fuzzy logic based controller, fuzzy logic takes rolling angle error and rate of change of error as input and generate voltages to control dc motor output accordingly. In this case, even though fluctuations decreased but ship stabilization time is quite long. Whereas in proposed technique, we used fuzzy logic to tune the PID controller initial gains according to rolling angle. This initial gain tuning increases the system efficiency as low initial fluctuation and stabilization time can be observed in Figure 14

Furthermore, the response of ship roll angle can be seen in Figure 15 without ARG, with passive and active ARG.

As we can see in Figure 14, ARG response with proposed fuzzy tuned PID controller is much faster and settling time is quite low without any big fluctuations.

\section{Hardware Implementation Method}

A practical ARG system consists of a high mass flywheel mounted on a motor, which rotates it to produce stabilization torque. This motor and flywheel is attached to a gimbal which allows it to rotate around its axis to provide precession angle 


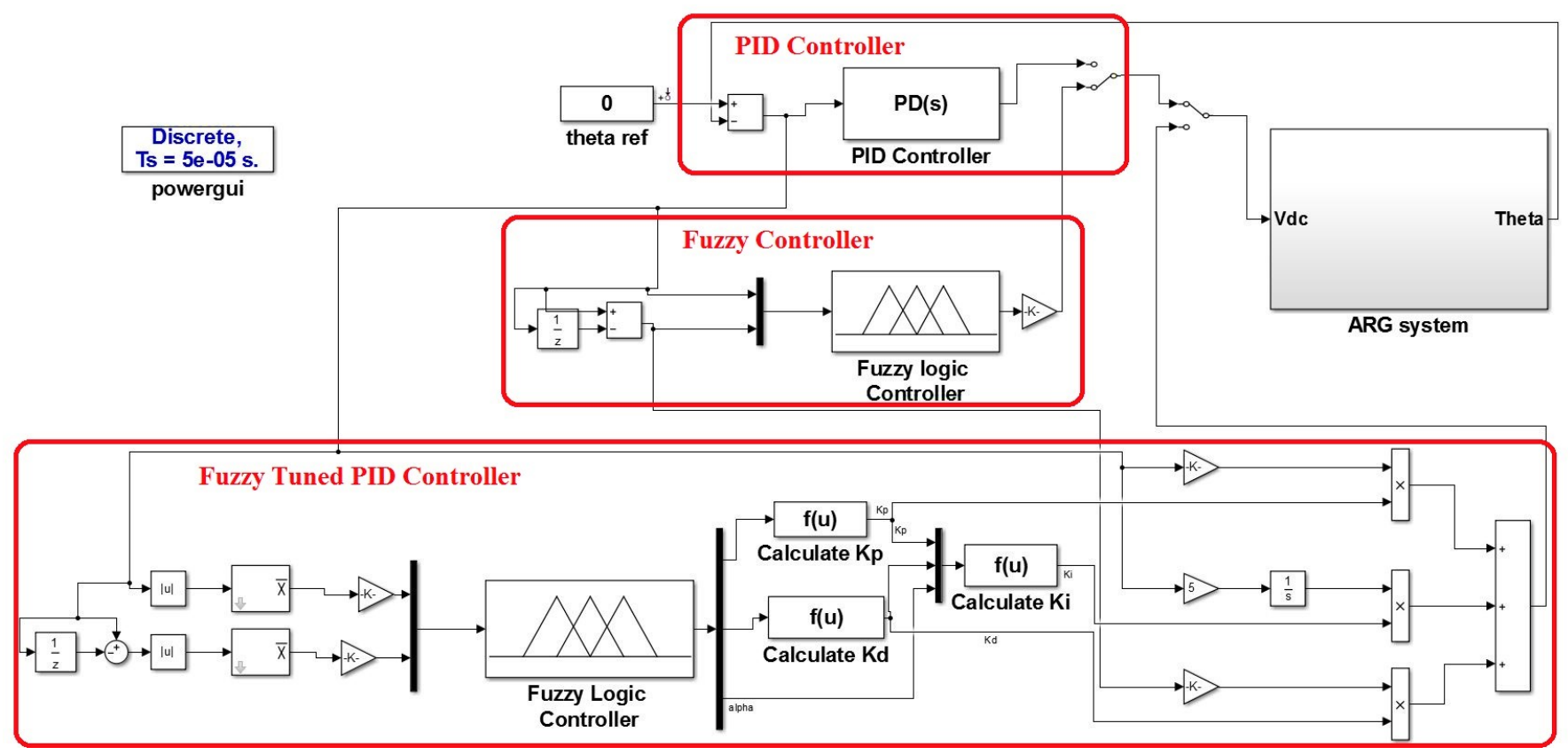

Figure 13. Designed system Simulink diagram.

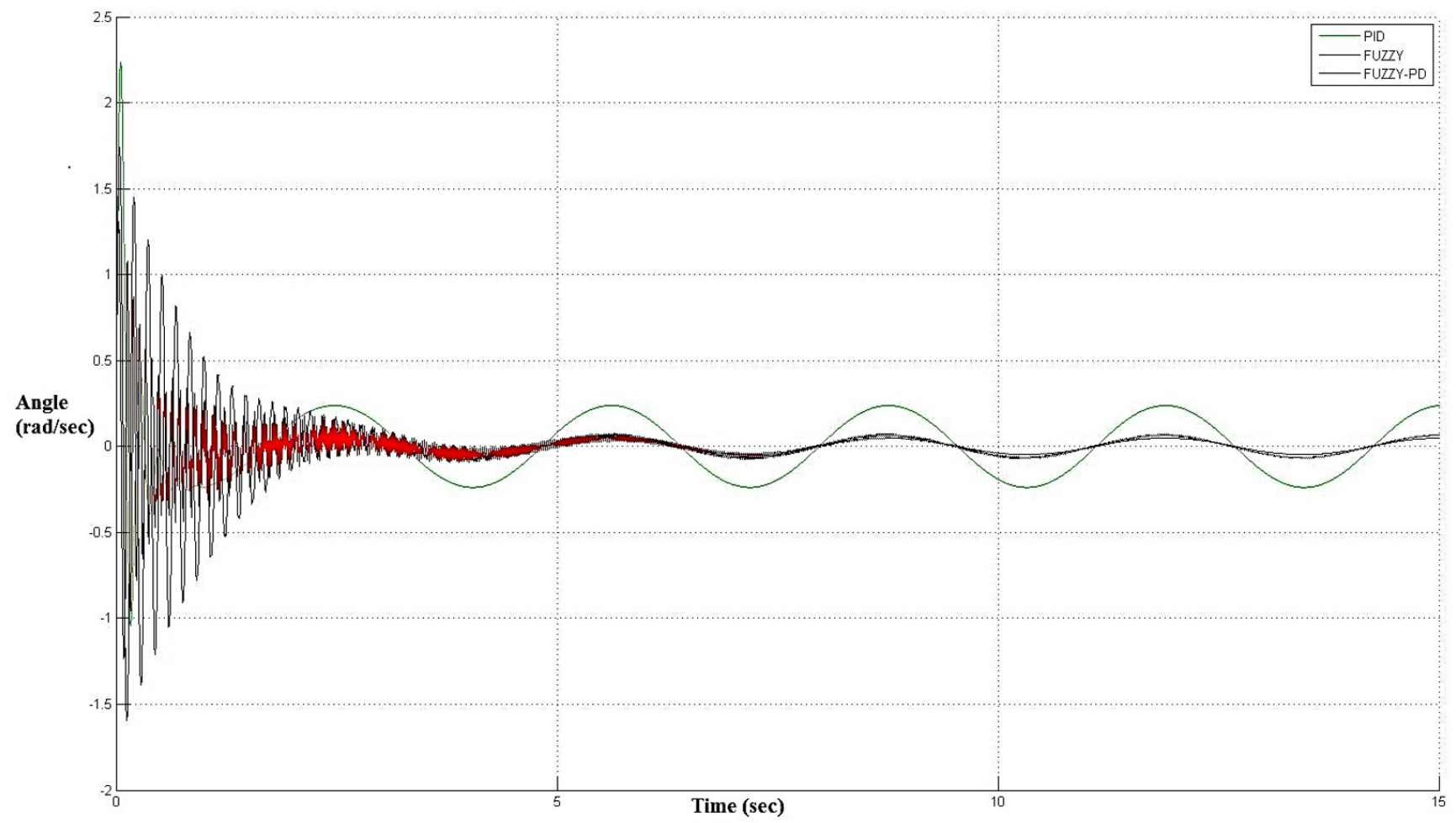

Figure 14. Comparison of proposed ARG stabilizing controller with previous techniques.

$\left(\alpha_{\text {prec }}\right)$ and precession velocity $\left(\omega_{\text {prec }}\right)$. Figure 16 explains the actual ARG construction. Passive ARG systems have simple bearings with a damper used as gimbal; whereas, active ARG systems consists of an additional motor attached to it for con- trol operation. The controller controls the angular velocity and direction of the gimbal via motor to provide exact amount of precession torque in correct direction. Gyro sensor and accelerometer are attached to the ship to sense roll angle and 


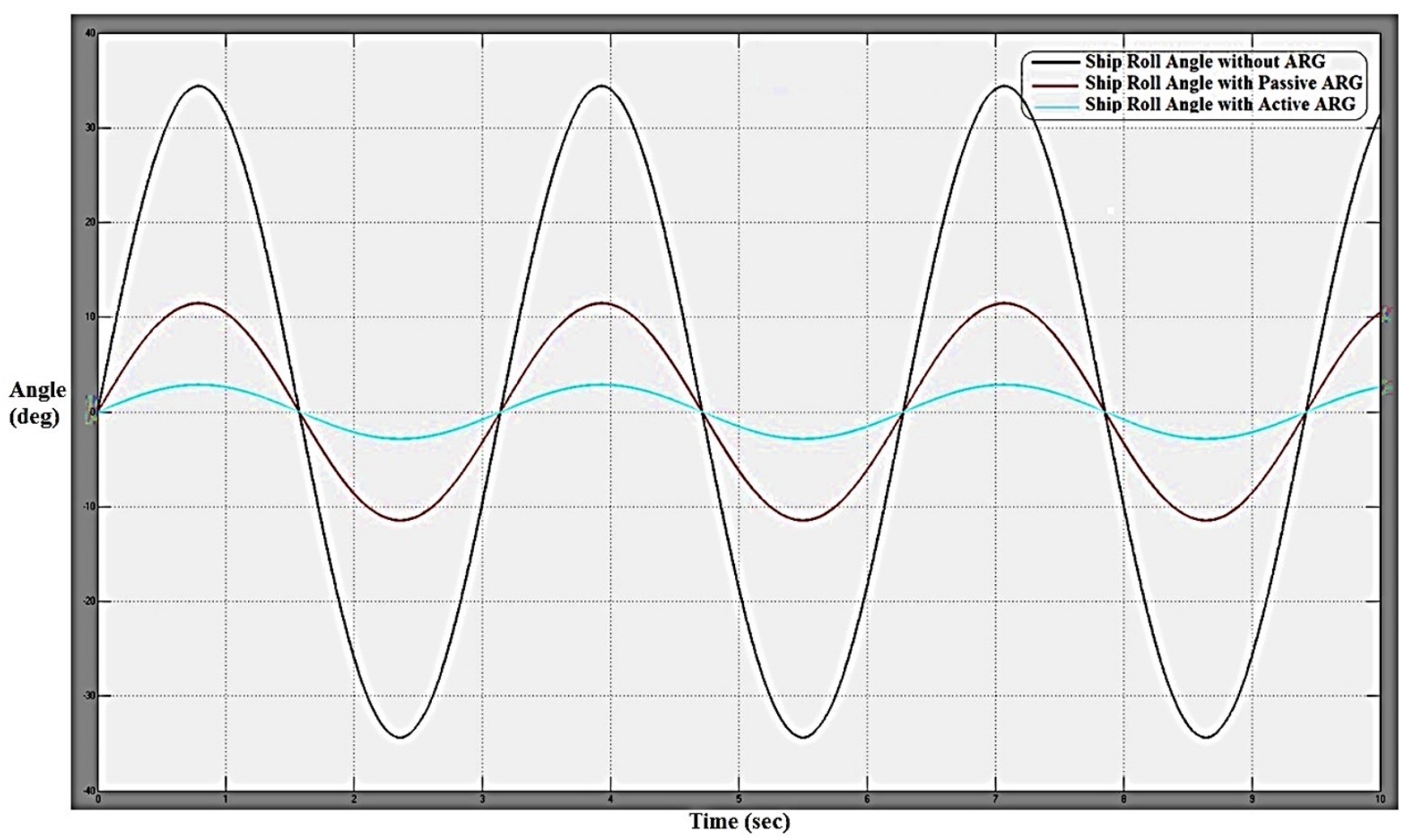

Figure 15. Ship roll angle response.

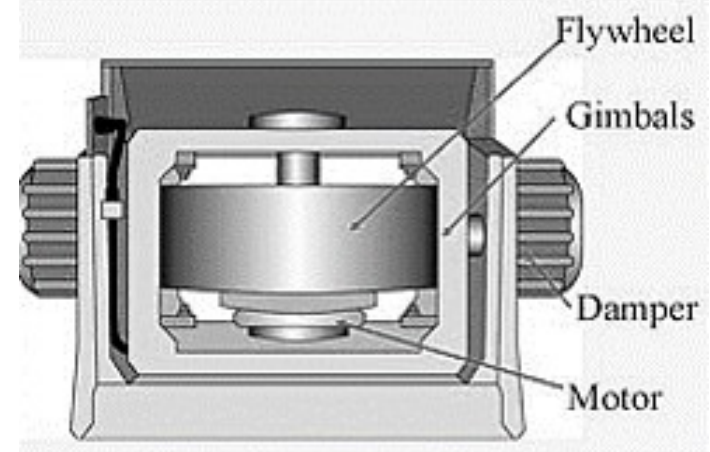

Figure 16. ARG system practical implementation overview.

controller performs control operation accordingly.

\section{Conclusion}

Robust and effectual controller for ARG stabilizer for ships has been proposed in this work. Fuzzy logic is used for gain tuning of PID controller for better response of ARG to stabilize the vessel. Comparison with previous techniques is also carried out. All mentioned control techniques were implemented in the Simulink environment. PID gain tuning by fuzzy logic shows the better response of controller in terms of low fluctuations and stabilizing time as compared to previously used methods. This study validates the feasibility and robust capabilities of the proposed controller for ARGs in ship stability.

\section{Conflict of Interest}

No potential conflict of interest relevant to this article was reported.

\section{References}

[1] G. Colvin, "Development and validation of control moment gyroscopic stabilization," Ph.D. dissertation, Department of Mechanical and Aerospace Engineering, The Ohio State University, Columbus, OH, 2014.

[2] T. I. Fossen, Guidance and Control of Ocean Vehicles. New York, NY: John Wiley \& Sons, 1994.

[3] T. I. Fossen, Marine Control Systems: Guidance, Navigation, and Control of Ships, Rigs, and Underwater Vehicles. Trondheim: Marine Cybernetics, 2002. 
[4] T. I. Fossen, Handbook of Marine Craft Hydrodynamics and Motion Control. New York, NY: John Wiley \& Sons, 2011.

[5] T. Perez, Ship Motion Contro: Autopilots with Rudder Roll Stabilisation and Combined Rudder-Fin Stabilisers. Heidelberg: Springer, 2005.

[6] E. V. Lewis, Principles of Naval Architecture. Vol. I: Stability and Strength. Alexandria, VA: Society of Naval Architects and Marine Engineers, 1988.

[7] E. V. Lewis, Principles of Naval Architecture. Vol. III: Motions in Waves and Controllability. Alexandria, VA: Society of Naval Architects and Marine Engineers, 1988.

[8] T. Perez and M. Blanke, "Ship roll damping control," Annual Reviews in Control, vol. 36, no. 1, pp. 129-147, 2012.

[9] J. M. J. Journee and J. Pinkster "Introduction in ship hydrodynamics," Lecture MT519, Delft University of Technology, The Netherlands, 2002.

[10] A. Hoshio, "Stabilizer by control moment gyroscope," Mitsubishi Juko Giho, vol. 31, no. 3, pp. 213-216, 1994.

[11] H. Takeuchi, K. Umemura, and S. Maeda, "Development of the Anti Rolling Gyro 375T (rolling stabilizer for yachts) using space control technology," Mitsubishi Heavy Industries Technical Review, vol. 48, no. 4, pp. 70-75, 2011.

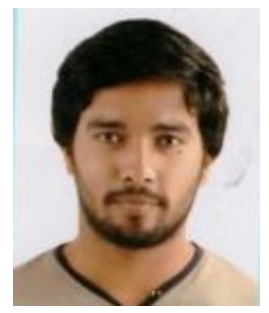

Muhammad Talha received the B.S. degree in Electrical Engineering from The University of Faisalabad in 2012. Finished M.S. in Control System Engineering from Kunsan National University in 2015. Current Ph.D. student in Kunsan National University. His research interests include renewable energy systems, power converters, and fuzzy logic.

E-mail: engr.talha72@gmail.com

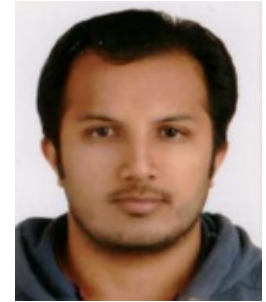

Furqan Asghar received the B.S. degree in Electrical Engineering from The University of Faisalabad in 2012. Finished M.S. in Control System Engineering from Kunsan National University in 2015. Current Ph.D. student in Kunsan National University. His research interests include renewable energy systems, artificial neural network, microgrid frequency and voltage stability and fuzzy logic.

E-mail: furqan@kunsan.ac.kr

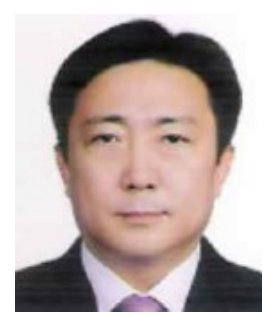

Sung Ho Kim received the B.S. degree in Electrical Engineering from Korea University in 1984. Finished M.S. from Korea University in 1986. Completed Ph.D. from Korea University in 1991. Completed POST-DOC from Hiroshima University (Japan). Current, Professor at Kunsan National University. His research interests include wind turbine system, sensor networks, neural network and fuzzy logic, intelligent control systems.

E-mail: shkim@kunsan.ac.kr 